\title{
Protective effect of resveratrol against acute lung injury induced by lipopolysaccharide via inhibiting the myd88-dependent Toll-like receptor 4 signaling pathway
}

\author{
ZHUO ZHANG ${ }^{1}$, NI CHEN ${ }^{2}$, JIN-BO LIU ${ }^{3}$, JIAN-BO WU ${ }^{2}$, JING ZHANG $^{4}$, YING ZHANG $^{4}$ and XIAN JIANG ${ }^{4}$ \\ ${ }^{1}$ Department of Pharmacology and ${ }^{2}$ Research Centre of Medicine and Functional Foods, \\ Luzhou Medical College; Departments of ${ }^{3}$ Clinical Laboratory and ${ }^{4}$ Anesthesiology, The First \\ Affiliated Hospital of Luzhou Medical College, Luzhou, Sichuan 646000, P.R. China
}

Received July 11, 2013; Accepted February 25, 2014

DOI: $10.3892 / \mathrm{mmr} .2014 .2226$

\begin{abstract}
Resveratrol, a polyphenol mainly present in grapes and red wine, demonstrated varied pharmacological activities. The protective effects of resveratrol on acute lung injury (ALI) induced by lipopolysaccharide (LPS) have remained elusive. The present study investigated whether the protective effect of resveratrol on ALI induced by LPS was via inhibiting the myeloid differentiation primary response gene (myd88)-dependent toll-like receptor (TLR)4 signaling pathway. Mice were pretreated with 5 or $45 \mathrm{mg} / \mathrm{kg}$ resveratrol for three days prior to bronchial perfusion with $25 \mathrm{mg} / \mathrm{kg}$ LPS. At $12 \mathrm{~h}$ after surgery, the ratio of the wet to the dry (w/d) lung was calculated to assess tissue edema. Histological changes of the lungs were detected using hematoxylin and eosin staining and the protein expression levels of TLR4, myd88 and nuclear factor kappa-light-chain-enhancer of activated $\mathrm{B}$ cells $(\mathrm{NF}-\kappa \mathrm{B})$ were measured by western blot analysis. The concentration of interleukin (IL)-6 and cyclooxygenase (COX)-2 in the bronchoalveolar lavage fluid were detected by ELISA. The results showed that resveratrol can suppress the edema, inflammatory cell infiltration and alveolar structure damage of lungs in mice with ALI and decrease the lung w/d ratio. Additionally, resveratrol markedly decreased the expression of TLR4, myd88 and $\mathrm{NF}-\kappa \mathrm{B}$ and decreased the concentration of inflammatory cytokines, including IL-6 and COX-2. Therefore, it can be concluded that resveratrol has a protective effect against ALI induced by LPS, at least in part by inhibiting the myd88-dependent TLR4 signaling pathway. In conclusion, resveratrol pretreatment has a protective effect against LPS-induced ALI in mice, which indicates that resveratrol may serve as a potential therapeutic agent for treating ALI in the clinic.
\end{abstract}

Correspondence to: Professor Xian Jiang, Department of Anesthesiology, The First Affiliated Hospital of Luzhou Medical College, 339 Zhongshan Road, Luzhou, Sichuan 646000, P.R. China E-mail: jiangxiandoctor@yeah.net

Key words: resveratrol, lipopolysaccharide, acute lung injury, toll-like receptor 4

\section{Introduction}

Acute lung injury (ALI) is a serious clinical problem associated with high morbidity and mortality $(1,2)$. The mortality rate of ALI in the United States is $40 \%$ (3). ALI is characterized by a disruption of the endothelium and alveolar epithelial barriers involving increased microvascular permeability, followed by an onset of dyspnea, severe hypoxemia and pulmonary edema $(4,5)$. ALI can develop numerous devastating complications at later stages, including severe sepsis, severe trauma and ischemia/reperfusion injury (6).

The mechanisms underlying ALI remain to be fully elucidated. Lipopolysaccharide (LPS), also termed endotoxin, is a component of the cell wall of gram-negative bacteria and is a causative agent implicated in the pathogenesis of ALI (7). LPS is a specific ligand of toll-like receptor 4 (TLR4) and ALI is associated with the activation of TLR4 signaling pathways induced by LPS. Although TLR4 is essential for initiating the activation of innate defenses, excessive inflammation in response to prolonged activation can prove detrimental (8-10). TLR4 signaling pathways include myeloid differentiation primary response gene (myd88)-dependent and -independent pathways (11). LPS is a major stimulus for the release of excess production of inflammatory mediators, including cytokines, chemokines and adhesion molecules via activating the myd88-dependent TLR4 signaling pathways, which may further cause pulmonary damage leading to ALI (12).

There are no specific therapies for ALI; however, mechanical ventilation strategies and management remain supportive (13). Chinese herbal medicine has the advantage of having anti-inflammatory properties and its aspects are economical and fine; therefore, it is useful to identify effective drugs for the treatment of ALI in Traditional Chinese Medicine.

Resveratrol (3,4',5-trihydroxy-trans-stilbene; Rsv), a polyphenol mainly present in grapes and red wine, has numerous bioactivities, including the inhibition of tumor initiation, anti-inflammatory, lipid modification, anti-oxidative, neuroprotective and anti-aging effects (14-16). Resveratrol has received increasing attention and has been associated with its inhibition of the nuclear factor $\kappa$-light-chain-enhancer of activated $\mathrm{B}$ cells $(\mathrm{NF}-\kappa \mathrm{B})$ transcription factor $(17,18)$. 
The role of resveratrol on ALI via inhibiting TLR4 and inflammatory factors, including interleukin (IL)-6 and cyclooxygenase (COX-2) in the TLR4 signaling pathway, remains poorly defined. Therefore, the present study was designed to investigate whether oral administration of resveratrol could ameliorate ALI induced by LPS, and to analyze whether resveratrol has a protective effect via inhibiting the myd88-dependent TLR4 signaling pathway (Fig. 1). The protein expression of TLR4, myd88 and NF- $\mathrm{kB}$ was detected by western blot analysis, and levels of IL-6 and COX-2 were detected by ELISA.

\section{Materials and methods}

Reagents. Resveratrol $\left(\mathrm{C}_{14} \mathrm{H}_{12} \mathrm{O}_{3}\right.$; MW, 228.24; purity $\left.>98 \%\right)$ was purchased from Guangxi Changzhou Natural Products Development Co., Ltd. (Nanning, China). LPS (Sigma, St. Louis, MO, USA), rabbit anti-mouse TLR4, myd88, NF-кB and $\beta$-actin antibodies were obtained from Abcam (Cambridge, MA, UK), bicinchoninic acid (BCA) protein assay kit was obtained from Pierce Biotechnology, Inc., (Rockford, IL, USA). IL-6 and COX-2 ELISA detection kits were purchased from R\&D Systems (Minneapolis, MN, USA). Goat anti-rabbit antibody was purchased from Abcam (Cambridge, MA, UK).

Animals and housing conditions. A total of 144 adult balb/c mice (age, 10 weeks; weight, 20-26 $\mathrm{g}$ at the beginning of the experiment) obtained from Jianyang Dashuo Animal Science and Technology Co., Ltd (Jianyang, China) were used in this experiment. All the mice received human care according to the guidelines of the Local Institutes of Health Guide for The Care and Use of Laboratory Animals. In total, 10 mice were housed in one cage and in a climate-controlled room $\left(25^{\circ} \mathrm{C}, 55 \%\right.$ humidity and 12 -h light/darkness cycle), fed a standard laboratory diet and acclimated five days prior to the experiment. Experiments were performed between 9:00 am and 5:00 pm. The present study was approved by the ethics committee of the First Affiliated Hospital of Luzhou Medical College, (Luzhou, Sichuan, China).

Trial grouping. The mice were randomly divided into six groups, $\mathrm{n}=24$ per group. Group one was the normal group, group two the ALI model group, with bronchial perfusion by $2.5 \mathrm{mg} / \mathrm{kg}$ LPS, group three was the sham surgery group, which was infused with sterile saline instead of LPS and groups four and five were LPS $+45,5 \mathrm{mg} / \mathrm{kg}$ resveratrol, which were orally administrated resveratrol $(45,5 \mathrm{mg} / \mathrm{kg})$ everyday for three days prior to an LPS challenge. Group six was the dexamethasone (Dex) group, which was administered $10 \mathrm{mg} / \mathrm{kg}$ Dex by intraperitoneal (i.p.) injection as a positive control.

LPS-induced ALI model. Mice were anesthetized with $3 \%$ pentobarbital sodium $(30 \mathrm{mg} / \mathrm{kg}, 0.1 \mathrm{ml} / 10 \mathrm{~g}$, i.p.) and were mounted on a tablet. Disinfection and a neck midline incision exposed the trachea, and mice were bronchially perfused with $25 \mathrm{mg} / \mathrm{kg}$ LPS in $40 \mu \mathrm{l}$ sterile saline within $1 \mathrm{~min}$. The control mice were administered the same amount of sterile saline, disinfection and suture incision $(19,20)$.

Bronchoalveolar lavage fluid (BALF) collection, leukocyte count and classification. At $24 \mathrm{~h}$ after surgery, one third of the

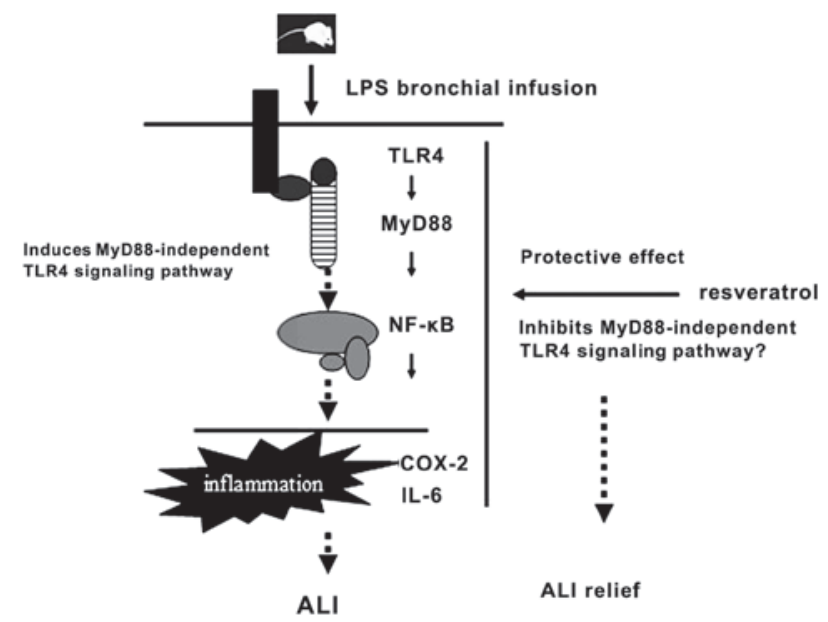

Figure 1. Hypothetic mechanism underlying the protective effect of resveratrol via inhibiting the myd88-dependent TLR4 signaling pathway. Myd88, myeloid differentiation primary response gene; TLR4, Toll-like receptor 4; COX-2, cyclooxygenase 2; IL, interleukin; NF- $\mathrm{kB}$, nuclear factor $\kappa$-light-chainenhancer of activated B cells; LPS, lipopolysaccharide; ALI, acute lung injury.

mice in each group were anesthetized with the same method as that in the aforementioned steps: Bronchus irrigation with saline at $0.5 \mathrm{ml}$ each time, continuously for five times, then BALF was obtained, and from half of the BALF, the red blood cells were removed by addition of red blood cell lysis buffer (21) and the sample was centrifuged at $250 \mathrm{x} \mathrm{g}$ for $10 \mathrm{~min}$. The supernatant was discarded and the pellet was suspended again in $1 \mathrm{ml}$ phosphate-buffered saline. The cells were stained for 5-10 min with Wright's stain at room temperature and different fields of view of 100 leukocyte cells were counted using optical microscopy. The mononuclear cells and lymphocytes were classified and their percentage was calculated. The remaining half was frozen at $4^{\circ} \mathrm{C}$ and the concentration of IL-6 and COX-2 was determined (22).

Lung wet-to-dry $(w / d)$ weight ratio. In total, $12 \mathrm{~h}$ following the LPS challenge, one third of the mice in each group were euthanized and the lungs were excised. Each lung was blotted dry, weighed, and then placed in an oven at $80^{\circ} \mathrm{C}$ for $48 \mathrm{~h}$ to obtain the 'dry' weight. The ratio of the wet lung to the dry lung was calculated to assess tissue edema (23).

Histological analysis. In total, $12 \mathrm{~h}$ after surgery, the mice were anesthetized with $3 \%$ pentobarbital sodium $(30 \mathrm{mg} / \mathrm{kg}$, $0.1 \mathrm{ml} / 100 \mathrm{~g}$; i.p.), perfused with formaldehyde, and the lung was removed and fixed in $10 \%$ neutral-buffered formalin for $24 \mathrm{~h}$ and subsequently embedded in paraffin. The sections were stained with hematoxylin and eosin (H\&E) using a standard protocol and analyzed by light microscopy (Olympus, Tokyo, Japan) (23).

Western blot analysis. The frozen lung samples were homogenized with $1 \%$ detergent lysis buffer, containing $50 \mathrm{mM}$ Tris- $\mathrm{HCl}$ (pH 7.5), $150 \mathrm{mM} \mathrm{NaCl}, 0.1 \%$ sodium dodecyl sulfate (SDS), $1 \%$ NonidetP-40, $0.5 \%$ sodiumdeoxycholate, $100 \mathrm{mg} / \mathrm{ml}$ phenylmethylsulfonyl fluoride and $1 \mathrm{mg} / \mathrm{ml}$ aprotinin. The protein concentrations were determined by a BCA protein assay kit. The protein extracts were fractionated by $12 \%$ SDS-PAGE and then 


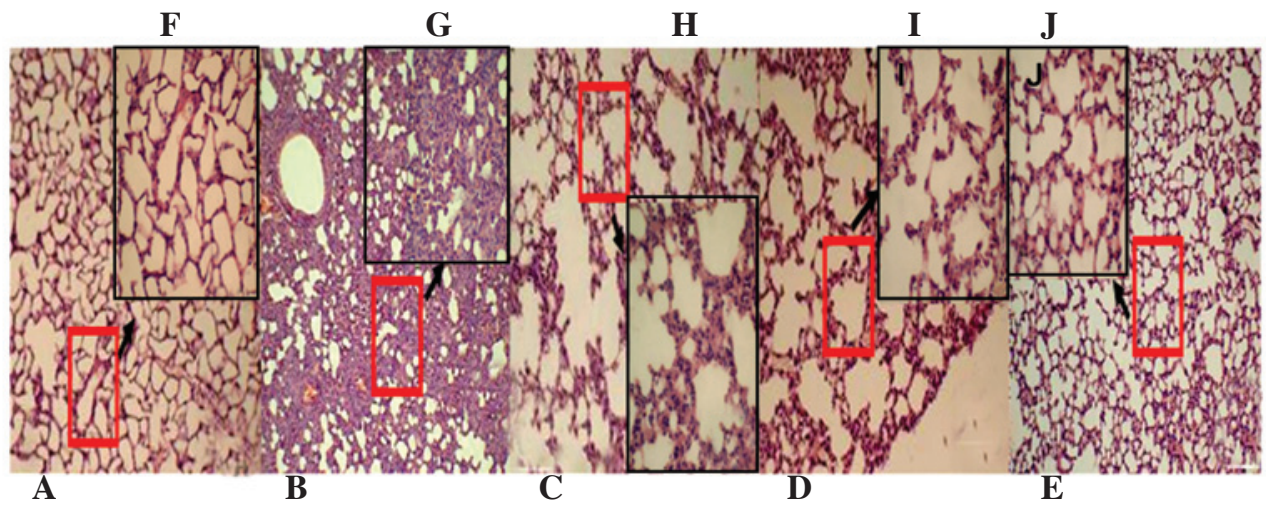

Figure 2. Effect of Rsv on histopathological changes in the lung. Tissues in ALI mice induced by LPS (A-E, magnification, x100; F-J, magnification, x200), The mice were given an oral administration of RSV ( 5 or $45 \mathrm{mg} / \mathrm{kg}$ ) every day for three days prior to administration of LPS. The lungs ( $\mathrm{n}=8$ ) from each experimental group were processed for histological evaluation at $12 \mathrm{~h}$ after the LPS challenge. (A,F) Control; (B,G) LPS; (C,H) Rsv (5 mg/kg); (D,I) Rsv (45 mg/kg) and (E,J) Dex $(10 \mathrm{mg} / \mathrm{kg})$ groups. Images shown here are representative slides from each group. ALI, acute lung injury; LPS, lipopolysaccharide; Dex, dexamethasone; Rsv, resveratrol.

transferred to a nitrocellulose membrane. The membrane was blocked with $5 \%(\mathrm{w} / \mathrm{v})$ skimmed milk in Tris-buffered saline containing $0.05 \%$ Tween 20 , followed by incubation with a rabbit anti-TLR4 $(1: 2,000)$, anti-myd88 $(1: 1,000)$ and anti-NF- $\kappa \mathrm{B}$ $(1: 1,000)$ antibody at $4^{\circ} \mathrm{C}$ overnight. Next, the membrane was treated with horseradish peroxidase-conjugated goat anti-rabbit secondary antibody $(1: 1,000)$ and antibody binding was visualized with an enhanced chemilluminescence reagent and short exposure to a Gel imaging system (Korda, Tokyo, Japan).

ELISA. The IL-6 and COX-2 concentration of BALF was quantified using a competitive enzyme immunoassay kit (R\&D Systems, Minneapolis, MN, USA) according to the manufacturer's instructions. All the experiments were performed in triplicate.

Statistical analysis. All the results are presented as the mean \pm standard deviation. Statistical analysis was performed with statistical analysis software SPSS 13.0 (SPSS, Inc., Chicago, IL, USA). Comparisons were performed using one-way analysis of variance in multiple groups, and the comparison between groups were performed using Student-Newman-Keuls test. $\mathrm{P}<0.05$ was considered to indicate a statistically significant difference.

\section{Results}

Resveratrol alleviated ALI induced by LPS in mice. To evaluate the effect of resveratrol on ALI, the histological changes of lungs in different groups were initially observed. The H\&E staining results revealed that $12 \mathrm{~h}$ after LPS administration in the model group, lung congestion, large amounts of leukocyte infiltration, an increase in the alveolar wall thickness, edema, hemorrhage and exudation in the alveolar cavity were present (Fig. 2B and G), which confirmed that the ALI model was established successfully. In comparison with the model group, the control group exhibited alveolar lobule structure integrity and alveolar clean cavity, without any edema in the alveolar walls (Fig. 2A and F). Pretreatment with resveratrol and Dex for three days can alleviate the changes of all the parameters mentioned in the model group (Fig. 2C, D, E, H, I and J).

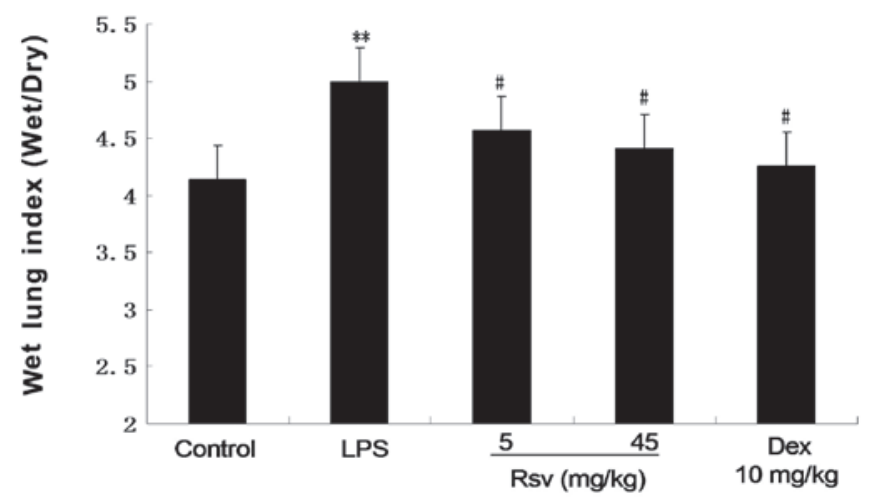

Figure 3. Effects of Rsv in the w/d ratio of the lungs. The mice were administered an oral dose of Rsv ( 5 or $25 \mathrm{mg} / \mathrm{kg}$ ) every day for three days prior to LPS challenge. The lung w/d ratio was determined at $12 \mathrm{~h}$ after the LPS challenge. The quantitative values presented are the mean \pm standard deviation $(\mathrm{n}=8) .{ }^{* *} \mathrm{P}<0.01$, compared with control group; ${ }^{\#} \mathrm{P}<0.05$, compared with LPS group. LPS, lipopolysaccharide; w, wet; d, dry; Dex, dexamethasone; Rsv, resveratrol.

Resveratrol attenuates the lung w/d ratio induced by LPS. The w/d ratio represents the degree of edema and inflammatory in the pulmonary system. The LPS challenge produced a significant increase in the pulmonary vascular permeability, and the w/d ratios of different groups were investigated further as shown in Fig. 3. The w/d ratio markedly increased in the LPS group, and compared with the LPS group, the w/d ratio of the Rsv group (5 or $45 \mathrm{mg} / \mathrm{kg}$ ) and the Dex (10 mg/kg) group was evidently decreased.

Resveratrol decreases the number and classification of leukocytes in BALF. Subsequent to obtaining the BALF, the red blood cells were removed with red blood cell lysis buffer and leukocytes isolated by centrifugation. In total, 100 leukocyte cells were counted by an optical microscope which indicated that the number of leukocytes and neutrophils markedly increased in the LPS group. In comparison with the LPS group, the number of leukocytes and neutrophils in the RSV group ( 5 or $45 \mathrm{mg} / \mathrm{kg}$ ) and the Dex group (10 mg/ $\mathrm{kg}$ ) was evidently decreased as shown in Fig. 4. 


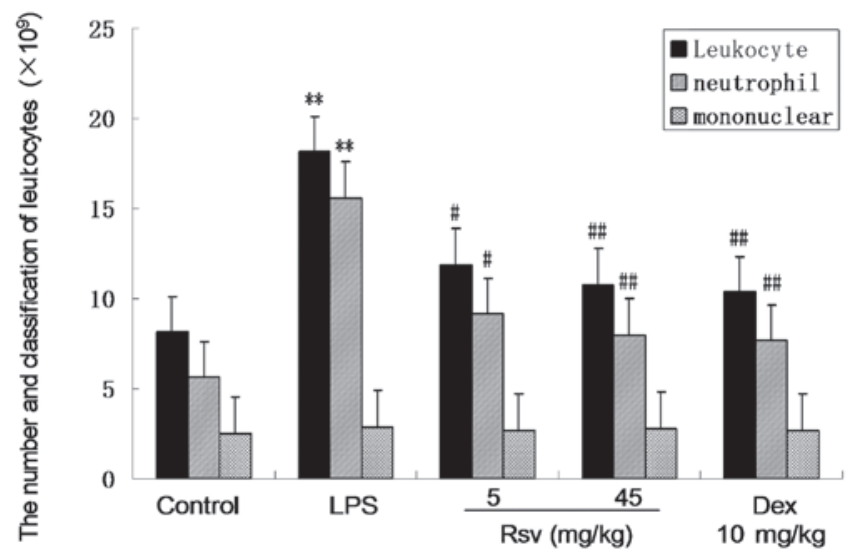

Figure 4. Number and classification of leukocytes in BALF. The mice were orally administrated with Rsv ( 5 or $25 \mathrm{mg} / \mathrm{kg}$ ) every day for three days prior to the LPS challenge. The number and classification of leukocytes in BALF was detected at $12 \mathrm{~h}$ after LPS challenge. The quantitative values presented are the mean \pm standard deviation $(\mathrm{n}=8) .{ }^{* *} \mathrm{P}<0.01$, compared with control group; ${ }^{\#} \mathrm{P}<0.01$ and ${ }^{\#} \mathrm{P}<0.05$, compared with the LPS group. BALF, bronchoalveolar lavage fluid; LPS, lipopolysaccharide; Dex, dexamethasone; Rsv, resveratrol.

Resveratrol reduces injury of the pulmonary system induced by LPS via inhibiting the protein expression of TLR4, myd88 and $N F-\kappa B$. The TLR4 signaling pathway serves as a molecular pattern recognition receptor associated with pathogens, which bind a microbial molecular motif with high affinity and have a central role in the initiation of cellular innate immune responses (24). LPS is a specific ligand of TLR4; therefore, the protein expression of TLR4, myd88 and NF- $\mathrm{B}$ was detected by western blot analysis. It was investigated whether the mechanism of ALI induced by LPS was associated with the myd88-dependent TLR4 signaling pathway. The results indicated that the protein expression of TLR4, myd88 and NF- $\kappa \mathrm{B}$ were markedly increased in the LPS group, while pretreatment with resveratrol can effectively suppress the protein expression of TLR4, myd88 and NF- $\mathrm{B}$ (Fig. 5A-D).

Resveratrol decreases the content of IL-6 in BALF of mice with LPS-induced ALI. IL-6 as a downstream factor of the TLR4 signaling pathway can reflect the severity of inflammation (25); therefore, the content of IL-6 in BALF was detected and the effect of resveratrol on ALI induced by LPS via inhibiting the IL- 6 was investigated. The results indicated that the content of IL-6 in BALF was markedly increased in the LPS group. In comparison with the LPS group, pretreatment with resveratrol or Dex can evidently decrease the content of IL-6 in BALF (Fig. 6A).

Resveratrol decreases the content of COX-2 in BALF of mice with LPS-induced ALI. COX-2 as a downstream factor of the TLR4 signaling pathway can reflect the severity of inflammation; therefore, the content of COX-2 in BALF was detected and the effect of resveratrol on ALI induced by LPS via inhibiting COX-2 was investigated. The results indicated that the content of COX-2 in BALF was markedly increased in the LPS group, and in comparison with pretreatment with resveratrol or Dex, the content of COX-2 in BALF was evidently decreased (Fig. 6B).
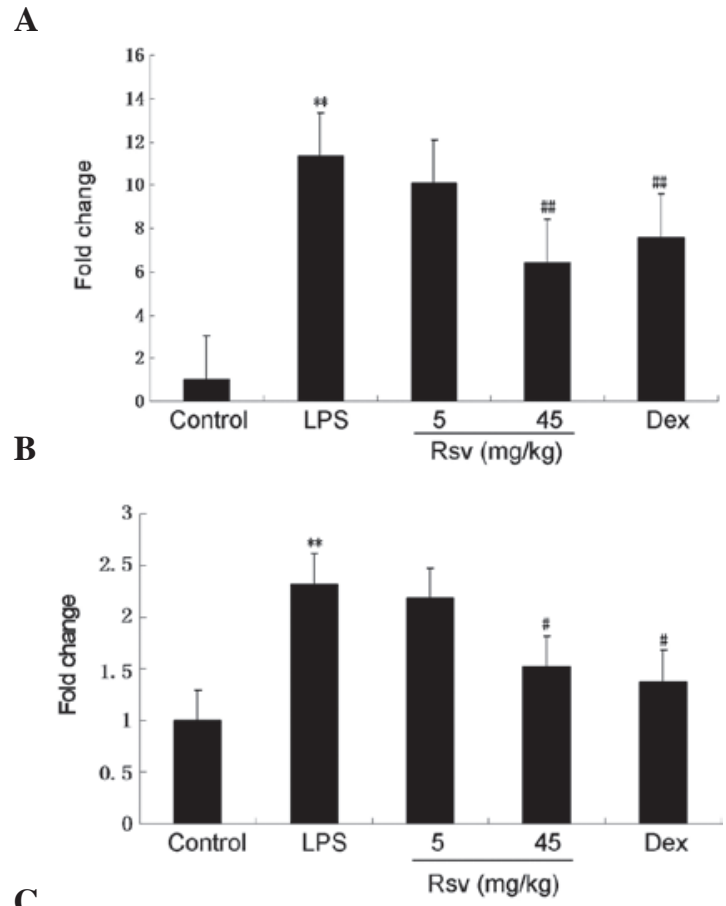

C

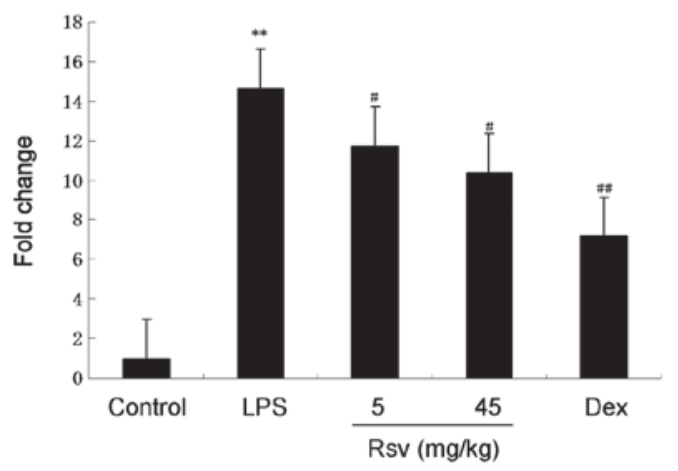

D

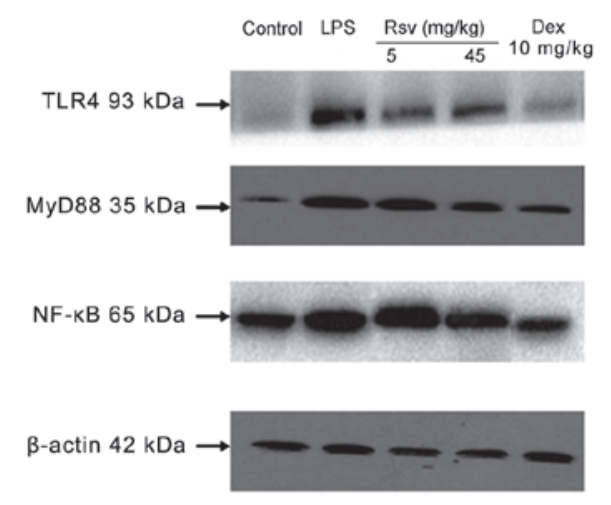

Figure 5. Pretreatment with Rsv inhibits TLR4, myd88 and NF- $\kappa$ B protein expression in the pulmonary system. (A) Fold-change of TLR4 expression; (B) fold-change of myd88 expression; (C) fold-change of NF- $\kappa \mathrm{B}$ expression and (D) western blot showing protein expression of TLR4, myd88 and $\mathrm{NF}-\kappa \mathrm{B}$; all in the pulmonary system. The mice were pretreated with Rsv ( 5 or $25 \mathrm{mg} / \mathrm{kg}$ ) every day for three days. Total protein extracts were prepared from the lung tissues $12 \mathrm{~h}$ after the LPS challenge, the expression

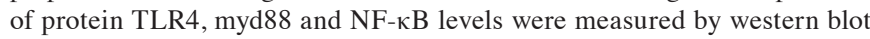
analysis. ${ }^{* *} \mathrm{P}<0.01$, compared with control group; ${ }^{\# \#} \mathrm{P}<0.01$, compared with LPS group; ${ }^{\mathrm{P}}<0.05$, compared with model group. The results shown here is one of three independent experiments. TLR4, Toll-like receptor 4; myd88, myeloid differentiation primary response gene $88 ; \mathrm{NF}-\kappa \mathrm{B}$, nuclear factor $\kappa$-light-chain-enhancer of activated B cells; LPS, lipopolysaccharide; Dex, dexamethasone; Rsv, resveratrol. 
A

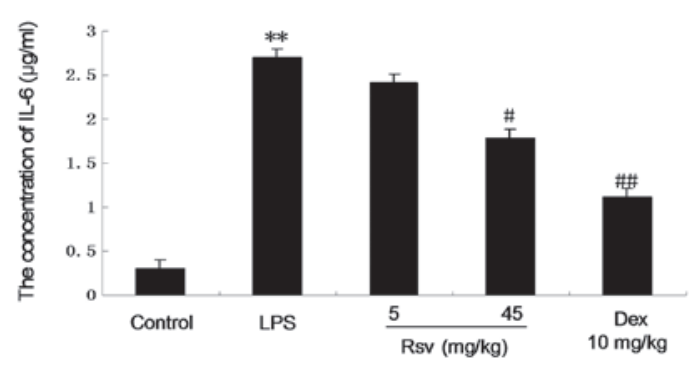

B

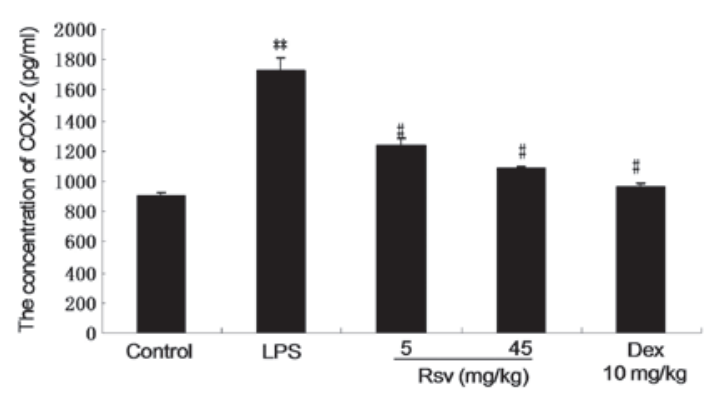

Figure 6. Rsv decreases the levels of (A) IL-6 and (B) COX-2 in the BALF of mice with ALI. The mice were pretreated with Rsv (5 or $25 \mathrm{mg} / \mathrm{kg}$ ) or Dex $(10 \mathrm{mg} / \mathrm{kg})$ every day for three days prior to LPS challenge. At $12 \mathrm{~h}$ after LPS treatment, BALF were collected and prepared for COX-2 leve determination. Data represent the mean \pm standard deviation of three independent experiments $(\mathrm{n}=8) .{ }^{* *} \mathrm{P}<0.01$, compared with control group; ${ }^{\# \#} \mathrm{P}<0.01$, compared with LPS group; ${ }^{\#} \mathrm{P}<0.05$, compared with model group. The result shown here is one of three independent experiments. IL, interleukin; COX-2, cyclooxygenase-2; BALF, bronchoalveolar lavage fluid; ALI, acute lung injury; Dex, dexamethasone; LPS, lipopolysaccharide; Rsv, resveratrol.

\section{Discussion}

Resveratrol is a chemical extract from traditional Chinese herbs and food. Resveratrol has diverse biochemical and physiological actions, including anti-cancer, anti-aging, anti-apoptosis and anti-inflammatory effects $(26,27)$. Previous studies have demonstrated that resveratrol exerts cardioprotective effects. In view of the anti-inflammatory effects of resveratrol, the present study has put forward the protective effects of resveratrol on LPS-induced ALI in mice which may be associated with TLR4 pathways. In the present study, an ALI model of mice was initially established via intratracheal instillation of LPS. Furthermore, the pathological changes of alveolar edema were observed through $\mathrm{H} \& \mathrm{E}$ staining and the protective effects of resveratrol on LPS-induced ALI in mice were analyzed. To further quantify the magnitude of the pulmonary edema, the lung w/d ratio was evaluated. Finally, the mechanism of the protective effect of resveratrol on LPS-induced ALI in mice was investigated and the protein expression of correlation factors, including TLR4, myd88, NF- $\mathrm{kB}$ and the concentration of inflammatory factors, including IL-6 and COX-2, which are involved in the myd88-dependent signaling pathway, were assessed. The principal findings of the present study were that: i) Pretreatment of resveratrol is able to attenuate edema, inflammatory cell infiltration and alveolar structure damage of the lungs in mice with ALI and significantly decrease the lung w/d ratio. ii) Resveratrol markedly decreased the protein expression of TLR4, myd88 and NF- $\mathrm{KB}$, and decreased the levels of inflammatory cytokines, including IL-6 and COX-2. It can therefore be concluded that resveratrol has a protective effect against ALI induced by LPS and is associated with the inhibition of the myd88-dependent TLR4 signaling pathway.

Previous studies have shown that ALI as well as the acute respiratory distress syndrome, which is known as a more severe form of ALI, are acute respiratory failure syndromes, which result from acute pulmonary edema and inflammation. ALI has several etiologies and the key mechanisms by which lung injury is initiated and propagated have yet to be defined. To date, the mortality syndrome of ALI is high $(28,29)$ and despite the fact that animal models provide a bridge between patients and the laboratory bench, the animal model of ALI remains incomplete as a model for the mechanisms and consequences of the disease in humans. In sepsis, LPS acts as a significant mediator in response to gram-negative bacteria. The classic model of ALI is a mouse model with ALI induced by intratracheal instillation of LPS, with its target being the capillary endothelium (30). Furthermore, LPS is known to be capable of entering the blood stream and eliciting inflammatory responses, which may lead to shock and mortality (31). In the present study, results for the LPS group indicated that $12 \mathrm{~h}$ after LPS administration, there was evident lung congestion, a high amount of leukocyte infiltration, an increased alveolar wall thickness, edema, hemorrhage and exudation in the alveolar cavity. Therefore, the ALI model in mice was successfully established.

TLR4 from humans and mice recognize different LPS structures. TLR4 has a significant role in the induction of the inflammatory response by recognition of several endogenous ligands associated with tissue injury (32). TLR4, in turn, activates the signaling pathways responsible for the generation of proinflammatory cytokines via myd88. Activation of TLR4 via myd88 induces NF-кB-dependent apoptosis and the expression of proinflammatory cytokines as a final step (33).

The proinflammatory cytokines appear in the early stages of an inflammatory response and may finally contribute to the severity of lung injury (34). Inflammatory factors may induce, enlarge and facilitate an entire or focal inflammatory reaction. TLR4 mediated NF- $\mathrm{\kappa B}$ activation and produced a large number of inflammatory cytokines (34). Numerous inducers are involved in the activation of NF- $\mathrm{KB}$, including the proinflammatory cytokines (mainly IL-6 and COX-2) and bacteria. IL-6 and COX-2 are considered pivotal mediators of lung inflammation in ALI as they can stimulate the production of a variety of chemokines and active neutrophiles (35). The key event involved in the activation of NF- $\mathrm{KB}$ is its nuclear translocation and activated NF- $\mathrm{KB}$ may translocate from the cytoplasm into the nucleus $(34,35)$. The target cells, including epithelial and endothelial cells, can be triggered to produce additional mediators by the release of IL-6 from alveolar macrophages. As a consequence, the initial inflammatory response can be amplified and the NF- $\kappa \mathrm{B}$ signaling pathway is activated in the lung tissue.

To further investigate the molecular mechanism of the protective effect of resveratrol on LPS-induced ALI in mice, the protein expression of the correlation factors, including TLR4, myd88 and NF- $\kappa B$, in the myd88-dependent signaling pathway was detected. IL-6 and COX-2 were also detected in the BALF of mice with ALI. Pretreatment with resveratrol was found to be capable of inhibiting TLR4/NF- $\mathrm{KB}$ and regulating the expression of IL- 6 and COX-2 via the myd88-dependent TLR4 signaling pathway. 
In conclusion, the present study revealed that resveratrol is effective for protecting mice against LPS-induced ALI, and it may be associated with the suppression of the activation of the myd88-dependent TLR4 signaling pathway, which may subsequently lead to a decrease in levels of proinflammatory cytokines. However, the present study did not investigate the effect of resveratrol on the myd88-independent TLR4 or other signaling pathways, nor on other proinflammatory cytokines in these pathways. These are the aims of future studies. Considering that resveratrol is widely distributed in fruit and was proven to have only few side effects, resveratrol may be a potential therapeutic agent for treating ALI in the future.

\section{References}

1. Li T, Zhang J, Feng J, Li Q, Wu L, Ye Q, Sun J, Lin Y, Zhang M, Huang R, Cheng J, Cao Y, Xiang G, Zhang J and Wu Q: Resveratrol reduces acute lung injury in a LPS-induced sepsis mouse model via activation of Sirt1. Mol Med Rep 7: 1889-1895, 2013.

2. Cabrera-Benitez NE, Pérez-Roth E, Casula M, Ramos-Nuez A, Ríos-Luci C, Rodríguez-Gallego C, Sologuren I, Jakubkiene V, Slutsky AS,Padrón JM and Villar J: Anti-inflammatory activity of a novel family of aryl ureas compounds in an endotoxin-induced airway epithelial cell injury model. PLoS One 7: e48468, 2012.

3. Rubenfeld G, Caldwell E, Peabody E, Weaver J, Martin DP, Neff M, Stern EJ and Hudson LD: Incidence and outcomes of acute lung injury. N Engl J Med 353: 1685-1693, 2005.

4. Zhang XQ, Lv CJ, Liu XY, Hao D, Qin J, Tian HH, Li Y and Wang XZ: Genome-wide analysis of DNA methylation in rat lungs with lipopolysaccharide-induced acute injury. Mol Med Rep 7: 1417-1424, 2013.

5. Devaney J, Contreras M and Laffey JG: Clinical review: gene-based therapies for ALI/ARDS: where are we now? Crit Care 15: 224, 2011.

6. Martínez O, Nin N and Esteban A: Prone position for the treatment of acute respiratory distress syndrome: a review of current literature. Arch Bronconeumol 45: 291-296, 2009 (In Spanish).

7. Nagaoka K, Takahara K, Tanaka K, Yoshida H, Steinman RM, Saitoh S, Akashi-Takamura S, Miyake K, Kang YS, Park CG and Inaba K: Association of SIGNR1 with TLR4-MD-2 enhances signal transduction by recognition of LPS in gram-negative bacteria. Int Immunol 17: 827-836, 2005.

8. Wang X, Bi Z, Wang Y and Wang Y: Increased MAPK and $\mathrm{NF}-\kappa \mathrm{B}$ expression of Langerhans cells is dependent on TLR2 and TLR4, and increased IRF-3 expression is partially dependent on TLR4 following UV exposure. Mol Med Rep 4: 541-546, 2011.

9. Andonegui G, Zhou H, Bullard D, Kelly MM, Mullaly SC, McDonald B, Long EM, Robbins SM and Kubes P: Mice that exclusively express TLR4 on endothelial cells can efficiently clear a lethal systemic Gram-negative bacterial infection. J Clin Invest 119: 1921-1930, 2009.

10. Diaz JV, Brower R, Calfee CS and Matthay MA: Therapeutic strategies for severe acute lung injury. Crit Care Med 38 1644-1650, 2010.

11. Takeda K, Kaisho T and Akira S: Toll-like receptors. Annu Rev Immunol 21: 335-376, 2003.

12. Youn HS, Lee JY, Fitzgerald KA, Young HA, Akira S and Hwang DH: Specific inhibition of MyD88-independent signaling pathways of TLR3 and TLR4 by resveratrol: molecular targets are TBK1 and RIP1 in TRIF complex. J Immunol 175: 3339-3346, 2005.

13. Karpurapu M, Wang X, Deng J, Park H, Xiao L, Sadikot RT, Frey RS, Maus UA, Park GY, Scott EW and Christman JW Functional PU.1 in macrophages has a pivotal role in NF- $\kappa$ B activation and neutrophilic lung inflammation during endotoxemia. Blood 118: 5255-5266, 2011.

14. Zheng M, Chen R, Zhong H, Lin Q, Wang X, Zhao Z and Xie L: Side-effects of resveratrol in HepG2 cells: reduced pten and increased bcl-xl mRNA expression. Mol Med Rep 6: 1367-1370, 2012.

15. Baur JA, Pearson KJ, Price NL, Jamieson HA, Lerin C, Kalra A, Prabhu VV, Allard JS, Lopez-Lluch G, Lewis K, Pistell PJ, Poosala S, Becker KG, Boss O, Gwinn D, Wang M, Ramaswamy S, Fishbein KW, Spencer RG, Lakatta EG, Le Couteur D, Shaw RJ, Navas P, Puigserver P, Ingram DK, de Cabo R and Sinclair DA: Resveratrol improves health and survival of mice on a high-calorie diet. Nature 444: 337-342, 2006.
16. Kim SY, Park KC, Kwon SB and Kim DS: Hypopigmentary effects of 4-n-butylresorcinol and resveratrol in combination. Pharmazie 67: 542-546, 2012

17. Athar M, Back JH, Tang X, Kim KH, Kopelovich L, Bickers DR and Kim AL: Resveratrol: a review of preclinical studies for human cancer prevention. Toxicol Appl Pharmacol 224: 274-283, 2007.

18. Kim MY: Nitric oxide triggers apoptosis in A375 human melanoma cells treated with capsicin and resveratrol. Mol Med Rep 5: 585-591, 2012.

19. Li W, Dai S, An J, Li P and Chen X: Chronic but not acute treatment with caffeine attenuates traumatic brain injury in the mouse cortical impact model. Neuroscience 151: 1198-1207, 2008.

20. Li J, Zhao L, He X, Zeng YJ and Dai SS: Sinomenine protects against lipopolysaccharide-induced acute lung injury in mice via adenosine A(2A) receptor signaling. PLoS One 8: e59257, 2013.

21. Rogerio AP, Fontanari C, Borducchi E, Keller AC, Russo M, Soares EG, Albuquerque DA and Faccioli LH: Anti-inflammatory effects of Lafoensia pacari and ellagic acid in a murine model of asthma. Eur J Pharmacol 580: 262-270, 2008.

22. Frenzel J, Gessner C, Sandvoss T, Hammerschmidt S, Schellenberger W, Sack U, Eschrich K and Wirtz H: Outcome prediction in pneumonia induced ALI/ARDS by clinical features and peptide patterns of BALF determined by mass spectrometry. PLoS ONE 6: e25544, 2011.

23. Li J, Zhao L, He X, Zeng YJ and Dai SS: Sinomenine protects against lipopolysaccharide-induced acute lung injury in mice via adenosine A2A receptor signaling. PLoS ONE 8: e59257, 2013.

24. Wang Y, He H, Li D, Zhu W, Duan K, Le Y, Liao Y and Ou YW: The role of the TLR4 signaling pathway in cognitive deficits following surgery in aged rats. Mol Med Rep 7: 1137-1142, 2013.

25. Zhou J, Zhang X, Zhang H, Jia Y, Liu Y, Tang Y and Li X: Use of data mining to determine changes in the gene expression profiles of rat embryos following prenatal exposure to inflammatory stimulants. Mol Med Rep 8: 95-102, 2013.

26. Bai Y, Mao QQ, Qin J, Zheng XY, Wang YB, Yang K, Shen HF and Xie LP: Resveratrol induces apoptosis and cell cycle arrest of human T24 bladder cancer cells in vitro and inhibits tumor growth in vivo. Cancer Sci 101: 488-493, 2010.

27. Kim MH, Yoo DS, Lee SY, Byeon SE, Lee YG, Min T, Rho HS, Rhee MH, Lee J and Cho JY: The TRIF/TBK1/IRF-3 activation pathway is the primary inhibitory target of resveratrol, contributing to its broad-spectrum anti-inflammatory effects. Pharmazie 66: 293-300, 2011.

28. Hou HW, Li XG, Yan M, Hu ZQ and Song YE: Increased leukocyte Rho-kinase activity in a population with acute coronary syndrome. Mol Med Rep 8: 250-254, 2013.

29. Wheeler AP and Bernard GR: Acute lung injury and the acute respiratory distress syndrome: a clinical review. Lancet 369: 1553-1564, 2007.

30. Teng P, Li YH, Cheng WJ, Zhou L, Shen Y and Wang Y: Neuroprotective effects of Lycium barbarum polysaccharides in lipopolysaccharide-induced BV2 microglial cells. Mol Med Rep 7: 1977-1981, 2013.

31. Knapp S, Florquin S, Golenbock DT and van der Poll T: Pulmonary lipopolysaccharide (LPS)-binding protein inhibits the LPS-induced lung inflammation in vivo. J Immunol 176: 3189-3195, 2006.

32. Bachmaier K, Toya S, Gao X, Triantafillou T, Garrean S, Park GY, Frey RS, Vogel S, Minshall R, Christman JW, Tiryppathi C and Malik AB: E3 ubiquitin ligase Cblb regulates the acute inflammatory response underlying lung injury. Nat Med 13: 920-926, 2007.

33. Shen Y, Wang Q, Zhao Q and Zhao J: Leptin promotes the immune escape of lung cancer by inducing proinflammatory cytokines and resistance to apoptosis. Mol Med Rep 2: 295-299, 2009.

34. Medvedev AE, Lentschat A, Wahl LM, Golenbock DT and Vogel SN: Dysregulation of LPS-induced Toll-like receptor 4-MyD88 complex formation and IL-1 receptor-associated kinase 1 activation in endotoxin-tolerant cells. J Immunol 169: 5209-5216, 2002.

35. Ware LB and Matthay MA: The acute respiratory distress syndrome. N Engl J Med 342: 1334-1349, 2000. 